\title{
Ethics Consultation for Mrs. Hamadani - A Focus on Process
}

\author{
Anita J. Tarzian
}

\section{Introduction}

My appreciation for process in ethics consultation was taken up a notch after experiencing how much thought our neighbors to the North put into procedural elements of professional practice. In 2011, I was privileged to attend a meeting of Canada's "Practicing Healthcare Ethicists Exploring Professionalization" (PHEEP) group in Saint John, New Brunswick. ${ }^{1}$ Much of that meeting was spent attending to how the newly-formed group would work together. While some of the Canadians bemoaned their commitment to how things are done as much as to what is accomplished, I remember making a "note to self" along the lines of: Attending mindfully to the process of how health care ethics consultation is done is just as important as demonstrating that ethics consultants have requisite skills and knowledge.

My work chairing the American Society for Bioethics and Humanities' (ASBH's) Core Competencies Update Task Force (Tarzian \& the ASBH Core Competencies Update Task Force, 2013), and my membership on ASBH's Quality Attestation Presidential Task Force, left me with an appreciation for procedural standards in health care ethics consultation (HCEC). Decades ago, when new ethical dilemmas and uncertainties borne out of medical innovations emerged on the front lines of health care delivery, and bewildered clinicians looked for someone to help them sort through the various ethical questions that arose, identifying what specific steps those "helping" such clinicians should follow was not a priority. The focus was more aptly situated on the ethical analyses applied to address uncertainty about the

\footnotetext{
${ }^{1}$ Now the Canadian Association of Practicing Healthcare Ethicists - Association canadienne des éthiciens en soins de santé (CAPHE-ACESS).

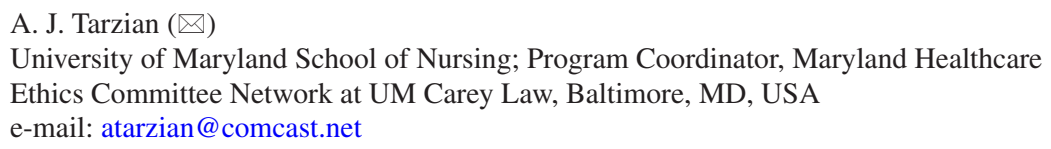


right decision or course of action to take in particular situations. Decades later, in a new century, when ethics consultation is available in most U.S. hospitals and is legislated in some states, the landscape has changed. We now have a code of ethics for HCE consultants which, among other things, ${ }^{2}$ obligates HCE consultants to "Be competent," to "Manage conflicts of interest and obligation," to "Contribute to the field," and to "Promote just health care within HCEC." Meeting these professional responsibilities requires active peer review. Toward that end, I'm grateful for the opportunity to provide my observations regarding Finder's involvement in the Hamadani ethics consultation. ${ }^{3}$

\section{Was This an Ethics Consultation?}

The first question is, did Finder provide an ethics consultation, or another ethics service in his role as health care ethics (HCE) consultant? The Core Competencies for Healthcare Ethics Consultation ("Core Competencies" ASBH 2011, p. 3) defines an ethics consultation as a "response to questions from patients, families, surrogates, health care professionals, or other involved parties who seek to resolve uncertainty or conflict regarding value-laden concerns that emerge in health care." HCE consultants can provide other services in addition to ethics consultation, such as ethics "coaching" and ethics education outside of what occurs during HCEC, overseeing an ethics consultation service, engaging in research or quality improvement initiatives, publishing scholarly work, mentoring other ethics consultants or interns, or providing proactive ethics services (Chidwick et al. 2010). It appears that not only was Finder's help solicited directly to address a question involving values conflicts, but Moore had also been asked to intervene by staff_-both during this hospital stay and prior hospital stays - to help resolve perceived conflicts between Mrs. Hamadani's children and the staff members caring for her. Thus, I do think this falls under the definition of ethics consultation, which is relevant in how Finder's performance is evaluated.

\footnotetext{
${ }^{2}$ The code of ethics responsibility to "Respect privacy and maintain confidentiality" requires either modifying the details of published case summaries so that those involved cannot be recognized, or obtaining permission to publish the summary of a case in which those involved might be recognizable. I have confirmed that this standard has been met for the Hamadani case, and I appreciate the book editors' attention to meeting this professional standard.

${ }^{3}$ Just as an ethics case consultation requires collecting information from involved parties to identify relevant contextual issues related to the case, an analysis of the HCE consultant's performance in a given case consultation likewise requires having access to contextual details. Finder has provided a rich recounting of the facts surrounding Mrs. Hamadani's final hospital stay and conflicts that arose between Mrs. Hamadani's children and her health care providers. Yet, a caveat of this performance evaluation is that information was not gathered first-hand. For example, I did not review any of Finder's notes written about this ethics consultation, nor speak first-hand with any of the involved parties. Thus, what I can provide here is an analysis of Finder's performance as ethics consultant based solely on the information he provided.
} 


\section{Case Consultation or Something Else?}

The next question is whether this constituted an ethics case consultation, i.e., an ethics consultation in which the HCE consultant interacts with a patient and/or the patient's family members. If the answer is "yes," then we would expect to see documentation of the HCEC in the patient's medical record. Other types of ethics consultations (referred to as "non-case consultations" in the Core Competencies) typically involve working solely with health care staff (e.g., helping staff debrief or process a difficult patient care situation, or helping them consider the ethical implications of a new institutional policy). Given Finder's interactions with Mrs. Hamadani's children, this seems aptly characterized as a case consultation. But there is a descriptive and a normative question here: was the consultation request handled as a case consultation, and should it have been handled as a case consultation?

Let's work backwards to consider Dr. Moore's involvement, since that influenced how Finder became involved. In a prior hospitalization, Dr. Moore responded to an ethics consultation request from a neurologist to address concerns that the Hamadani family was inappropriately requesting life-prolonging treatments for Mrs. Hamadani that increased her suffering without appreciably benefitting her. There appear to have been concerns about how the Hamadani children interacted with staff, and perhaps some concern with how decisions were being made for Mrs. Hamadani. The Core Competencies (2011) encourages each institution to have an identified process for how its ethics consultation service will handle HCEC requests. That way, consultations can be evaluated based on whether they meet institution-based standards (which should meet field-wide HCEC standards). Initially, the consultant should clarify the request. As stated in the Core Competencies (ASBH 2011: 12):

Generally this requires considering the preliminary information received at the time of the request, confirming that the request is appropriate for ethics consultation, setting reasonable expectations with the requester about what the ethics consultant will and will not do, and developing an initial formulation of the ethics question(s) that will be addressed by the consultation.

Presumably, Dr. Moore confirmed that the staff member requesting the initial consultation preferred that Dr. Moore treat this as an ethics case consultation, which would involve Dr. Moore speaking directly with various stakeholders - including with Mrs. Hamadani (if able) and the Hamadani children-to gather information (alone if using a single-consultant model; in collaboration with other ethics consultants if using a duo or team model). This might have been followed by a meeting with staff (often first without and later with the Hamadani family), with Dr. Moore leading those meetings.

I think it would be appropriate to proceed with a case consultation approach if Dr. Moore believed this was the best way to meet the needs of those involved. Often, health care staff prefer such an approach because it frees up time they would otherwise have to spend communicating with various stakeholders, and they value the unbiased perspective of the ethics consultant(s). However, I also think it's valuable to offer options when they exist and to clarify the preferred role the ethics consultant 
is playing. For example, staff might value an opportunity to review with Dr. Moore how they have been communicating with the Hamadani family about goals of care and decision-making as her cancer spread and her disease progressed. The mentioned lack of anyone taking a lead in identifying what interventions, if any, were no longer appropriate to offer Mrs. Hamadani demonstrates a failure of leadership on the part of the attending physician that should be addressed. Dr. Moore could either offer to take on the traditional role of the ethics consultant facilitating communication across consulting services, or he could offer to work with the attending physician to identify better ways of ensuring that the staff are "all on the same page."

The difference here is mostly procedural, and I'll return to this when addressing Finder's involvement in the case. The point is that having a standard approach to ethics consultation does not mean that the ethics consultant must play the same role in each consultation-rather, the consultant should find out what the requestor wants help with and then clarify how the consultant proposes to help. For example, the ethics consultant could have offered to accompany staff when they met with members of the Hamadani family (alone or during care coordination meetings) to provide feedback on communication encounters. In this instance, rather than acting as "lead consultant" in facilitating a formal meeting with the patient/family and the health care staff, the ethics consultant would be playing the role of ethics expert by providing constructive feedback about how the staff are communicating with each other and with the Hamadani family. Or, if the central issue focused on the conflict over Mrs. Hamadani's resuscitation status, Finder may have played a mediation role. The importance is allowing for the role of the ethics consultant to match the needs of the requestor(s), and clarifying what role the ethics consultant is playing.

\section{T'aarof as Ethics?}

Finder wisely identified many cultural influences that complicated the Hamadani ethics consultation. I'm reminded of a medical Grand Rounds presentation I gave many years ago entitled, "Challenging the Golden Rule: The Influence of Culture in Healthcare Encounters." Along with other examples, I referenced the Persian concept of T'aarof, a ritual politeness code that governs behaviors between individuals of different hierarchies and imposes obligations to mitigate emotional distress by way of avoiding negative feelings through specific culturally-engrained social etiquette. An Iranian physician in the audience admitted to feeling overwhelmed by competing cultural and professional obligations when caring for his traditional Iranian patients. He asked me what he could do about this. It struck me that providing "culturally competent care" is a challenge not only when a clinician comes from a culture that is different from the patient's culture, but also when the clinician shares a culture with a patient that differs from mainstream U.S. medical culture. It's clear that this contributed substantially to the perceived conflicts between the Hamadani family and the staff caring for Mrs. Hamadani. What's less clear is the role that the ethics consultants played in addressing the ethical issues encountered by those involved. 


\section{The Role of the Ethics Consultant}

It's a poignant note that Samir, Mrs. Hamadani's son, first thought to ask Finder for help after seeing the title "Director of Ethics" on his name badge. From the description of how the interactions between the Hamadani family and hospital staff unfolded, it's likely that Samir's understanding of "ethics" and Finder's role as an ethics consultant differed from other staff members' perceptions. Despite some of the Hamadani children being more acculturated to the U.S. than others, there are several examples provided indicating that T'aarof may have influenced their expectations and led them to perceive that hospital staff had breached culturally-embedded ethical norms of conduct, Dr. Broukhim notwithstanding. Finder aptly identifies several of these norms: being a good listener, showing an interest and spending time getting to know family members, not distracting family away from the patient's bedside, the youngest son avoiding thinking about his mother's death to remain "strong" for his older sisters, and not focusing on what won't be done at the moment of death but on what it means to respect and care for one's mother leading up to and at the moment of her death.

Let's assume that Samir's expectation in asking for Finder's help was to reconcile a perceived breach of good conduct on the part of Dr. Moore. Is this a valid request for ethics consultation? While lodging a complaint about a "bad actor" often involves referring the requestor to the appropriate supervisor for follow up, in this case, I would argue that Samir's request is appropriate for ethics consultation, given the prior history and complex components of the case. Finder appropriately addresses the question of who should respond to this consult by concluding that one shouldn't force family members to talk with ethics consultants they don't want to talk to, and since Samir requested that Dr. Moore no longer speak with him or his sisters, Finder takes over Dr. Moore's role as the ethics consultant on this case. As Director of Ethics and thus Dr. Moore's supervisor, this simplifies Finder's decision to take over the case (since he presumably has the authority to do this).

Finder writes down some notes about his conversation with Samir, he reviews Mrs. Hamadani's chart, he talks with the intensive care unit (ICU) staff, and he meets with Dr. Broukhim. This is a good beginning. From this point on, however, there is ambiguity in several procedural aspects of how this consultation was handled. First, it wasn't entirely clear how limits on Dr. Moore's involvement would be communicated to other staff, as Finder stated to Moore: "I think it's OK if you want to keep talking with Broukhim since you've been helping him as much as anything. But really try not to put yourself in a position that the family might misperceive as your still being involved with them. I'll take over that" (Finder 2018: 28). Second, it's not clear that Finder has clarified what role he will play in this case. When meeting with Dr. Broukhim and Mrs. Hamadani's children, it's Dr. Broukhim who leads the discussion, leaving Finder to ponder how he should contribute. It's Dr. Broukhim who suggests, in his chart notes, the kind of assistance Finder can offer, including to "provide support" to the family, to "delineate ethical issues" involving Mrs. Hamadani's care, "not to persuade or to be abrasive" but "just to provide more care 
for them in these trying times" (Finder 2018: 30). Dr. Broukhim explains to Samir that the meeting with Finder "is not about Dr. Moore or Ethics Consultation but only about his mother" (Finder 2018: 30). Finder wonders if Dr. Broukhim is looking for "reassurance that he was helping Mrs. Hamadani's children come to accept that he had few to no options left to provide for their mother" (Finder 2018: 34).

It would have been helpful if Finder had clarified his role with Dr. Broukhim before the first meeting with Samir, Nadira, and Farzana, as Finder admits: "I wasn't sure if I was there to help Dr. Broukhim or to facilitate a conversation, or both, or just to bear witness..." (Finder 2018: 38). Finder ultimately suggests that Dr. Broukhim play the role of communication broker, telling Samir, Nadira and Farzana that "all the communication about what's going to be done for your mother [can] flow through him" to keep everyone "on the same page" so that "what one person says is not misunderstood or confused by what others say or mean or intend" (Finder 2018: 41). Does Finder mean that he will assist Dr. Broukhim in communicating with other members of the treatment team? It's unclear. He goes on to tell Mrs. Hamadani's children that the "real issue" is making sure that they understand what's going on and that their "expectations match" to avoid the problem of not being "prepared for what's staring us in the face." Again, it's unclear what this means, exactly. Who really is in charge, and how is Finder helping resolve this ambiguity?

\section{Influence of Various Cultural Orientations}

Just as Mrs. Hamadani and her children come from a particular cultural orientation, members of her treatment team come from their own personal and professional cultural orientations. Dr. Broukhim's orientation is influenced both by his professional training and his Persian roots. He spends many hours with his patients, referring to his "respect" and "devotion" toward his patients and their family members, writing detailed notes in their charts, and offering oncology treatments that some of his colleagues believe go "too far" in promoting "false hopes." Finder hints at the professional cultural norms in Western acute care and oncology settings. For example, it's clear that most of the staff caring for Mrs. Hamadani believed that when metastatic cancer is inoperable and advanced, ICU-level care and related interventions (such as intubation and ventilation, renal dialysis, and cardio-pulmonary resuscitation attempts) cause more harm than benefit and in some instances should not be offered. There are several references to staff focusing on interventions that should be withheld or withdrawn, as evident in Dr. Moore's advice to Finder: "I'd just warn you that they may be using you, to avoid what we'd already agreed upon in terms of talking about why they want to code her and have the feeding tube and whatever other aggressive interventions that may be available" (Finder 2018: 28).

While Finder recognizes these cultural differences in his write-up of "The Zadeh Scenario," it's unclear whether or how he addressed these conflicts in his role as the ethics consultant. It seems even Dr. Broukim questions whether he goes too far in what he offers to his patients. For example, he asks Finder whether dialysis would be reasonable for Mrs. Hamadani. Finder replies that while there are those who 
would consider dialysis in this case to be "a bad use of a limited resource" and "a prime example of why our healthcare system is in the state it's in and why we need reform," that if the goal of care is to give Mrs. Hamadani "a few more days," a few rounds of dialysis could help (Finder 2018: 35). Is this guidance sufficient to address the ethical conflicts this case presents?

\section{Mixed Messages}

Given that Mrs. Hamadani cannot communicate her wishes and there's no mention of an advance directive, medical decision-making is shared between her children and the attending physician (it's implied that Dr. Broukhim is the attending physician; the ICU attending's role is unclear). Finder assumes that Mrs. Hamadani's children are making choices based on what she would have wanted and/or on her best interests. What choices are available to them depends on the medical standard of care. This is where additional ambiguity clouds the ethics consultant's role. What medical interventions should be available to Mrs. Hamadani-and patients as sick as her-is an open question. The staff believed Mrs. Hamadani's children were making bad decisions and causing their mother to suffer. Finder uses the word "futile" to describe how staff felt about the use of aggressive life-prolonging interventions for Mrs. Hamadani (Finder 2018: 23). But he doesn't address which proposed interventions, if any, might actually fall into that category. The physicians involved in this case appear to disagree about what medical interventions were appropriate for Mrs. Hamadani. For example, Dr. Smith and Dr. Nadouri do not consider dialysis to be helpful in achieving "long-term goals" (Finder 2018: 31).

The role of the ethics consultant in such a case should be to help reduce this ambiguity by facilitating communication and educating those involved about standards for decision-making and for resolving conflicts. For example, White and Wicclair (2012) proposed three categories of medical interventions: generally accepted, generally unaccepted, and a middle category comprising the "grey zone." Grey zone interventions are often at the center of ethics consultations. White and Wicclair proposed that within the grey zone, clinicians should identify "medically inadvisable" interventions that should be withheld or withdrawn and should use a fair process for resolving disagreements. These are interventions that might accomplish an effect that a patient or family member desires, but that could be withheld based on the following ethical justifications: (1) they are unlikely to achieve their intended goal, (2) the goal they are intended to achieve is of controversial value, or (3) they are not cost-effective. This is consistent with a more recent policy statement on "potentially inappropriate treatment" developed by the Society of Critical Care Medicine's ethics committee (Kon et al. 2016).

Dr. Broukhim recommends that Mrs. Hamadani forego cardio-pulmonary resuscitation (CPR) attempts, as this "will not change the inevitability that awaits her" and "will likely cause her harm" (Finder 2018: 40). He communicates this to her children, but also tells them that if they wished their mother to be "coded" then he would "support them" (Finder 2018: 40). This is a mixed message. Similarly, he 
provides the children with vivid depictions of their mother's deteriorating physical status and impending death, but then reassures them that he is not trying to "change their mind about what to do" or getting them to "say that they should withhold additional interventions or even withdraw any of the support currently being provided" (Finder 2018: 39). Perhaps like the Iranian physician in my Grand Rounds presentation, Dr. Broukhim struggles with what T'aarof demands in these situations. It clearly results in confusion regarding what interventions Mrs. Hamadani can receive. It's also unclear to the Hamadani children and perhaps to Dr. Broukhim himself that their decision-making is shared, but that ultimately, the attending physician writes medical orders, as Mrs. Hamadani's children believe they can decide themselves whether their mother is intubated or whether CPR is attempted (when in fact these are medical orders). Why didn't Finder point out these contradictions to Dr. Broukhim? Why not assemble the treatment team and facilitate a discussion about how judgments should be made about which treatments are considered medically inappropriate, and where to draw the line with what should be offered to Mrs. Hamadani and similarly situated patients?

\section{Ambiguity Regarding Ethically Appropriate Interventions}

Finder's involvement with Mrs. Hamadani originated from Samir's dissatisfaction that Dr. Moore and the staff were over-focusing on a Do-Not-Resuscitate (DNR) order for his mother. Finder alludes to — but doesn't explicitly articulate—-the actual ethical issues he identifies after talking to all those involved. Staff are angry and resentful toward Mrs. Hamadani's children for making their mother suffer and for being overly demanding and at times intimidating; some are upset with Dr. Broukhim and Dr. Spector for offering interventions that appeared to meet White and Wicclair's definition of "medically inadvisable." This contributes to the conflict between Mrs. Hamadani's children and the treatment team, as evidenced by Samir exclaiming: "I do not need to hear 'we do not want your mother to suffer.' What I want is for my mother to be taken care of, and when the time comes to make a decision, my sisters and I will decide, and we will do what is best for our mother" (Finder 2018: 41).

The difficulty identifying which "grey zone" interventions qualify as "medically inadvisable" rests on judgments about which goals for Mrs. Hamadani are of "controversial value." The placement of a G-tube or naso-gastric tube is easier to classify as inadvisable for someone so close to death. Dialysis is a bit more difficult. A clinical practice guideline from the Renal Physicians Association (RPA 2010) recommends that dialysis not be provided to patients like Mrs. Hamadani who are near death from non-renal causes. Yet, the contested ICU transfer, blood pressure stabilizers, Bi-pap mask, and renal dialysis were implemented to achieve a goal of improving Mrs. Hamadani's mental status such that she could interact with her children one last time. Is this a worthwhile goal? Dr. Broukhim surmises: "it's not unreasonable to see if we can wake her up and give them a chance to have a little bit of final time together. She's been a real fighter all along, and they've been so 
involved in her care, I think I owe them this" (Finder 2018: 35). One may argue that this is an ethically justifiable goal, particularly given the likelihood that Dr. Broukhim has led Mrs. Hamadani's children to believe that her "fighting" to live is of value and something they should encourage. Dr. Broukhim would not be the first oncologist to interpret success as keeping his patients alive, while overlooking the value (and duty) of effectively preparing patients and their loved ones for death (hence, Samir's extreme reaction to the mention of hospice). He also would not be the first oncologist who needs support accepting his own patient's death and guidance in how to help families do the same (Graham 2013).

What's missing is a strategy for determining how these decisions are made in a way that minimizes regrets for the survivors, meets fairness criteria, and addresses the moral residue and distress of the staff. Mrs. Hamadani's children have asked that they speak with no other doctor but Dr. Broukhim — an unrealistic expectation, and perhaps one that Dr. Broukhim himself might have appreciated being liberated from with Finder's help. Nurses are refusing to care for Mrs. Hamadani. Samir "fires" the neurologist for mentioning hospice and Dr. Moore for talking about resuscitation status. These dynamics bode poorly for providing effective end-of-life care-either to Mrs. Hamadani or to others who are subject to the same staff communication breakdowns. Being a good listener is necessary-but not sufficient—for being an effective ethics consultant.

\section{Conclusion}

Despite the several potential shortcomings outlined above regarding how Finder handled the Hamadani case consultation, all was not lost. Just as an ounce of prevention is worth a pound of cure, a pound of active listening breeds ample good will. Finder facilitated good communication with Mrs. Hamadani's children and with Dr. Broukhim. He realized that discussing Mrs. Hamadani's resuscitation status or dialysis with Samir, Nadira or Farzana would only thwart future communication with them, so he simply listened. He picked up on the importance of spending time with them and asking them about their family. As far as his involvement with Samir, Nadira, Farzana, and Dr. Broukhim goes, this may fit within what I call a "mosaic" approach: even if parts are missing, there's enough there to see the whole picture. That is, even if Mrs. Hamadani's care was challenged by conflicting opinions and staff resentment, she died in a way that was consistent with how her family wished for her to be treated. Presumably, Samir, Nadira, and Farzana felt supported in the end.

It's unclear, however, whether the rest of the staff involved in this case felt supported by Finder's involvement. I wonder what would have happened if Finder had met with the treatment team after his initial involvement and facilitated a discussion about Mrs. Hamadani's care, her children's behavior, Iranian culture, and standards for judging interventions as "medically inadvisable." That way, a plan could have been constructed for how to provide respectful end-of-life care to Mrs. Hamadani without crossing ethical, professional, and emotional boundaries. Sometimes staff 
angst is grounded in frustration about the lack of consensus on where boundary lines are drawn, and poor communication about short- and long-term goals of care for a particular patient. The example of Mrs. Hamadani remaining a "full code" is a good one. Writing a DNR order over Samir's and Farzana's objections would have caused them distress and may have complicated their grieving. At the same time, the thought of having to attempt CPR for Mrs. Hamadani likely exacerbated the staff's distress. Here we have a classic conflict over positions. Could there be common interests underlying these competing positions? This is clearly the realm of the ethics consultant, but I didn't see where these ethics facilitation skills were applied to this case.

\section{References}

American Society for Bioethics and Humanities (2011) Core competencies for healthcare ethics consultation, 2nd edn. American Society for Bioethics and Humanities, Glenview

Chidwick P, Bell J, Connolly E, Couglin MD, Frolic A, Hardingham L, Zlotnik SR (2010) Exploring a model role description for ethicists. HEC Forum 22(1):31-40

Finder SG (2018) The Zadeh scenario. In: Finder SG, Bliton MJ (eds) Peer review, peer education, and modeling in the practice of clinical ethics consultation: the Zadeh project. Springer, Cham, pp 21-42

Graham J (2013) When the doctor disappears. The New York Times. Available at: http://newoldage.blogs.nytimes.com/2013/11/14/when-the-doctor-disappears/?_r=0. Last accessed 2 Nov 2014

Kon, A. et al. (2016). Defining futile and potentially inappropriate interventions: A Policy statement from the Society of Critical Care Medicine Ethics Committee. Crit Care Med, 44, 1769-17.

Renal Physicians Association (2010) Shared decision-making in the appropriate initiation of and withdrawal from dialysis, 2nd edn. Clinical Practice Guideline. Renal RPA, Rockville

Tarzian AJ, The ASBH Core Competencies Update Task Force (2013) Health care ethics consultation: an update on core competencies and emerging standards. AJOB 13(2):3-13

White DB, Wicclair M (2012) Limits on clinicians' discretion to unilaterally refuse treatment. Am J Crit Care 21(5):361-364

Open Access This chapter is licensed under the terms of the Creative Commons Attribution 4.0 International License (http://creativecommons.org/licenses/by/4.0/), which permits use, sharing, adaptation, distribution and reproduction in any medium or format, as long as you give appropriate credit to the original author(s) and the source, provide a link to the Creative Commons license and indicate if changes were made.

The images or other third party material in this chapter are included in the chapter's Creative Commons license, unless indicated otherwise in a credit line to the material. If material is not included in the chapter's Creative Commons license and your intended use is not permitted by statutory regulation or exceeds the permitted use, you will need to obtain permission directly from the copyright holder. 